Arq. Bras. Med. Vet. Zootec., v.66, n.3, p.927-932, 2014

\title{
Frequência de alimentação para juvenis de tambaqui
}

\author{
[Feeding frequency for tambaqui juveniles] \\ R.C. Souza ${ }^{1}$, D.F.B. Campeche $^{2}$, R.M.L. Campos $^{1}$, R.A.C.R. Figueiredo ${ }^{3}$, J.F.B. Melo ${ }^{1}$ \\ ${ }^{1}$ Universidade Federal do Vale do São Francisco - Univasf - Petrolina, PE \\ ${ }^{2}$ Pesquisadora Embrapa Semiárido - Petrolina, PE \\ ${ }^{3}$ Codevasf

\section{RESUMO}

Objetivou-se nesta pesquisa avaliar qual frequência alimentar promove o melhor desempenho em juvenis de tambaqui. Foram testadas quatro frequências alimentares (duas, quatro, seis e oito vezes ao dia) em intervalos regulares. Foram estocados alevinos de tambaqui com peso médio inicial de $15,74 \pm 0,28 \mathrm{~g}$ em 16 aquários de vidro transparentes com capacidade de $70 \mathrm{~L}$ de volume útil, em um delineamento inteiramente ao acaso, com quatro tratamentos e quatro repetições. O período de alimentação foi de 30 dias, e utilizou-se uma ração comercial contendo $45 \%$ de proteína bruta para peixes onívoros, com taxa de arraçoamento de $6 \%$ da biomassa total dos peixes de cada aquário. Os resultados médios de $\mathrm{pH}$, temperatura $\left({ }^{\circ} \mathrm{C}\right)$ e condutividade elétrica $(\mathrm{mS} / \mathrm{cm})$ para manhã e tarde foram, respectivamente: 6,91; 6,96; 25,85; 27,69; 0,65; e 0,65. Foram avaliados os índices de desempenho: ganho de peso, conversão alimentar aparente, taxa de crescimento específico, sobrevivência, e também foi analisada a glicose sanguínea. Não foram detectadas diferenças significativas para as variáveis estudadas. Recomenda-se a frequência de alimentação de duas vezes ao dia para juvenis de tambaqui, principalmente visando minimizar mão de obra.

Palavras-chave: Colossoma macropomum, desempenho, manejo alimentar, nutrição de peixes, peixe nativo

\begin{abstract}
The aim of this research was to assess how often food promotes better performance in tambaqui. We tested four food frequencies (two, four, six and eight times a day) at regular intervals. Tambaqui fingerlings with initial weight of $15.74 \pm 0.28 \mathrm{~g}$ were stocked in 16 transparent glass tanks with a capacity of 70 L of usable volume in a completely randomized design with four treatments and four replications. The feeding period was thirty days and a commercial diet containing $45 \%$ crude protein for omnivorous fish and a feeding rate of $6 \%$ of the total biomass of fish for each experimental unit was used. The average results of $\mathrm{pH}$, temperature ${ }^{\circ} \mathrm{C}$ and electrical conductivity $\mathrm{mS} / \mathrm{cm}$ for morning and afternoon were respectively 6.91 and $6.96 ; 25.85$ and 27.69; 0.65 and 0.65. We evaluated the performance indices: weight gain, feed conversion, specific growth rate, survival, and also blood glucose. No significant differences were detected for the variables studied. It is recommended that the frequency of feeding be 2 times a day for tambaqui, mainly to minimize labor.
\end{abstract}

Keywords: Colossoma macropomum, performance, alimentary handling, fish nutrition, native fish

\section{INTRODUÇÃO}

Alguns fatores podem ser afetados pela frequência alimentar, como espécie, idade do animal, temperatura da água (Hayashi et al.,
2004). Frascá-Scorvo (1999) cita outros aspectos importantes a serem considerados, como: anatomia, fisiologia, comportamento e hábito alimentar dos animais. Na maioria das pesquisas realizadas com diversas espécies e hábitos alimentares, podem-se encontrar animais que se

Recebido em 5 de abril de 2012

Aceito em 11 de fevereiro de 2012

E-mail: renildesouza@hotmail.com 
desenvolvem bem com uma, duas, três ou mais alimentações diárias (Hayashi et al., 2004; Ferreira et al., 2007; Graeff e Pruner, 2008; Corrêa et al., 2009; Corrêa et al., 2010). No tambaqui, especificamente, ainda não foi proposto um modelo de frequência de alimentação, e os existentes não são unânimes quanto à frequência utilizada. Desta forma, é necessário determinar a frequência ideal nas várias fases de criação, considerando-se as diferenças na idade dos animais, a composição das rações, a qualidade da água e as condições experimentais. Esses fatores podem variar até na mesma espécie (Sampaio et al., 2007) e nos diversos sistemas de cultivo.

O tambaqui é uma espécie natural da bacia amazônica, que possui um alto potencial de cultivo e grande importância econômica e social para as famílias ribeirinhas do Amazonas (Melo et al., 1998; Val et al., 2000), e constitui a principal espécie nativa produzida no Brasil (Paula, 2009). Por ser onívoro, com tendência à herbivoria, filtrador e frugívoro (Nunes et al., 2006), aceita facilmente rações artificiais, adapta-se bem ao cativeiro (Santos et al., 2010), além de apresentar boas características zootécnicas, como rápido crescimento e excelente ganho de peso. Contudo, ainda são escassos os trabalhos envolvendo o seu cultivo, principalmente relacionados à frequência de alimentação, à digestibilidade, à avaliação de alimentos e, ainda, ao conhecimento do perfil de metabólitos ou da atividade de enzimas, que, segundo Melo (2004), são ferramentas necessárias para entender o aproveitamento dos nutrientes, verificar situações metabólicas indesejáveis, pois constituem indicativos do estado nutricional dos animais.

Uma vez que a ração é um dos itens mais onerosos nos diversos sistemas de produção de peixes, com $50 \%$ do custo total (Andrade et al., 2005), é necessário adotar medidas que visem à redução deste custo, por meio de um adequado manejo alimentar. A implementação de uma frequência alimentar ótima, aliada a um alimento de qualidade, promove uma menor lixiviação dos nutrientes, reduzindo a carga poluente lançada no ambiente, além de otimizar a mão de obra. Considerando-se que o consumo de ração dos animais está diretamente relacionado com a saúde dos peixes, o ajuste pode prejudicar a conversão alimentar e, assim, constituir fonte de prejuízo ao produtor. Meer et al. (1997) afirmam que o excesso de alimento provoca alterações metabólicas, o que agrava a eficiência de absorção dos nutrientes.

Desta forma, objetivou-se neste trabalho determinar qual frequência alimentar promove o melhor desempenho em alevinos de tambaqui.

\section{MATERIAL E MÉTODOS}

O experimento foi conduzido nas instalações do Laboratório de Aquicultura da Universidade Federal do Vale do São Francisco. Foram utilizados 64 juvenis de tambaqui (Colossoma macropomum), com peso médio inicial de $15,74 \pm 0,28 \mathrm{~g}$, em um delineamento inteiramente ao acaso, com quatro tratamentos (duas, quatro, seis e oito vezes ao dia) e quatro repetições (Tab. 1). Utilizaram-se aquários com capacidade de 70L de volume útil, com entrada e saída de água individualizada, cada um com quatro alevinos.

Tabela 1. Número e horário das alimentações diárias dos alevinos de tambaqui

\begin{tabular}{ccccc}
\hline \multicolumn{5}{c}{ Frequência de alimentação diária } \\
\hline \multirow{4}{*}{ Manhã } & 2 vezes & 4 vezes & 6 vezes & 8 vezes \\
& $09: 00$ & $09: 00$ & $09: 00$ & $09: 00$ \\
& - & - & & $10: 00$ \\
Tarde & - & $11: 00$ & $10: 30$ & $11: 00$ \\
& - & - & $12: 00$ & $12: 00$ \\
& - & $13: 00$ & & $13: 00$ \\
& $15: 00$ & $15: 00$ & $15: 00$ & $15: 00$ \\
& - & - & & $16: 00$ \\
\hline
\end{tabular}

Os animais foram adaptados às unidades experimentais por um período de cinco dias, arraçoados três vezes ao dia. Foi adicionado nos aquários $0,1 \%$ de cloreto de sódio $(\mathrm{NaCl})$ como medida profilática, segundo as recomendações de Kubitza (2007).

A qualidade da água foi monitorada por meio de oxigenação constante por compressor de ar. A temperatura e o $\mathrm{pH}$ eram aferidos diariamente: de manhã, às $8 \mathrm{~h} 30 \mathrm{~min}$, e à tarde, às $15 \mathrm{~h} 30 \mathrm{~min}$, sempre antes do arraçoamento. Após realização das medidas de qualidade da água, os aquários eram sifonados para retirada de fezes e eventuais sobras de ração, com troca de $50 \%$ da água em 
cada sifonagem. A alimentação dos animais foi feita utilizando-se ração comercial contendo $45 \%$ de proteína bruta, para peixes onívoros de até $60 \mathrm{~g}$. O arraçoamento foi feito a uma taxa de $6 \%$ da biomassa total. O período experimental foi de 30 dias.

Ao final do período experimental, todos os peixes foram pesados, e imediatamente coletouse sangue mediante punção no vaso caudal com seringas contendo anticoagulante (Chagas et al., 2005). Logo após a coleta de sangue, os animais foram insensibilizados com gelo com posterior sacrifício para tomada de dados de desempenho. As variáveis avaliadas foram: ganho médio de peso final, conversão alimentar aparente, taxa de crescimento específico, sobrevivência e glicose sanguínea.

Os dados coletados foram submetidos à análise de variância e ao teste Tukey para comparação entre as médias com nível de significância de $5 \%$, utilizando-se o programa estatístico ASSISTAT 7.5 beta.

\section{RESULTADOS E DISCUSSÃO}

Os resultados médios de qualidade da água, $\mathrm{pH}$, temperatura $\left({ }^{\circ} \mathrm{C}\right)$ e condutividade elétrica $(\mu \mathrm{s} / \mathrm{cm})$, nos períodos da manhã e da tarde, foram, respectivamente: 6,$91 ; 6,96 ; 25,85 ; 27,69$;
0,65; e 0,65, e mantiveram-se dentro dos valores recomendados para a criação de peixes em clima tropical (Sipaúba-Tavares, 1995).

Os valores médios dos parâmetros de ganho de peso médio total, conversão alimentar aparente, taxa de crescimento específico, sobrevivência e glicose sanguínea estão apresentados na Tab. 2. Não foram encontradas diferenças significativas $(\mathrm{P}>0,05)$ entre os tratamentos para as variáveis estudadas. Segundo Luz e Portella (2005), a frequência de alimentação pode ou não afetar o desempenho dos animais. A frequência de duas vezes ao dia neste estudo seria suficiente para os dados avaliados. Em estudo com alevinos de jundiá, Carneiro e Mikos (2005), ao ofertarem ração uma, duas, três e quatro vezes ao dia, não encontraram diferenças significativas para ganho médio de peso, conversão alimentar aparente e taxa de crescimento específico, resultado semelhante aos observados no presente trabalho. Porém, estes autores destacam que, mesmo não encontrando diferenças significativas no desempenho dos animais, um aspecto importante a se considerar refere-se à observação do comportamento e da saúde dos animais. O mesmo fato ocorreu com híbridos de Clarias gariepinus, os quais apresentaram desempenho e crescimento satisfatórios com frequência de duas e três vezes ao dia (Ndome et al., 2011).

Tabela 2. Variáveis médias de desempenho e glicose dos alevinos de tambaqui submetidos a várias frequências de alimentação por um período de 30 dias

\begin{tabular}{cccccc}
\hline \multicolumn{5}{c}{ Frequência de alimentação diária } \\
\hline PMI (g) & 2 & 4 & 6 & 8 & CV $(\%)$ \\
GPMF (g) & 16,02 & 15,88 & 15,39 & 15,7 & 3,95 \\
CTRA (g) & 40,99 & 46,7 & 38,8 & 42,66 & 20,76 \\
CAA & 223,29 & 226,24 & 210,19 & 201,19 & 7,74 \\
TCE (\%) & 1,52 & 1,26 & 1,37 & 2,01 & 25,35 \\
GL (mg/dL) & 4,2 & 4,53 & 4,16 & 4,35 & 10,70 \\
S (\%) & 97,75 & 89 & 82,87 & 89,25 & 13,89 \\
\hline
\end{tabular}

PMI - peso médio inicial; GPMF - ganho de peso médio final; CTRA - consumo total de ração aparente; CAA conversão alimentar aparente; TCE - taxa de crescimento específico; GL - glicose plasmática; S - sobrevivência; $\mathrm{CV}$ - coeficiente de variação.

Em outro estudo com Colossoma macropomum, foram testadas duas taxas de alimentação (5 e
$10 \%$ do peso vivo), combinadas com duas frequências alimentares (duas e três vezes ao 
dia), e relatou-se que uma frequência de três vezes e uma taxa de $10 \%$ implicaram melhor desempenho dos animais (Silva et al., 2007). Ao testarem frequências alimentares de uma, duas e três vezes ao dia em tambaquis cultivados em tanques-rede, Corrêa et al. (2009) encontraram diferenças significativas no ganho de peso e na taxa de crescimento específico dos animais, quando, com, três alimentações diárias, obteve-se peso final superior. Sousa et al. (2012) verificaram que o aumento da frequência alimentar melhora o desempenho produtivo de tilápias-do-nilo produzidas em tanques-rede.

O consumo total de ração aparente não sofreu influência das frequências testadas. Meer et al. (1997) verificaram repostas diferentes em relação ao presente estudo; com cinco frequências alimentares diárias e ingestão voluntária em alevinos de tambaqui, aumentaram-se a ingestão de alimentos e a taxa de crescimento. Em contraponto, Kubitza (1997) sugere que altas frequências alimentares geralmente estão associadas a aumento na ingestão e podem aumentar a taxa de trânsito gastrointestinal, bem como prejudicar o desempenho. Neste trabalho, a maior frequência pode não ter atingido a máxima capacidade de consumo dos animais, pois a oferta era em pequenas quantidades. Além disso, o desempenho nos animais não alterou, o que pode estar relacionado ao consumo.

Assim como o consumo de ração, a conversão alimentar aparente não apresentou diferenças significativas $(\mathrm{P}>0,05)$. Este resultado foi semelhante ao encontrado em robalo-peva submetido às diferentes frequências alimentares (Corrêa et al., 2010). Da mesma forma, Ferreira et al. (2007), ao avaliarem uma, quatro e seis frequências diárias em Salminus brasiliensis, não encontraram diferença significativa na conversão alimentar aparente, porém recomendaram oferta de alimentação aos animais seis vezes ao dia, baseados na taxa de crescimento específico e na sobrevivência, que foram superiores. Fiod et al. (2010), ao analisarem a frequência alimentar de uma, duas e três vezes ao dia para pacu (Piaractus mesopotâmicus), não observaram diferenças para a conversão alimentar aparente.

A taxa de crescimento específico não foi influenciada pelas frequências testadas neste trabalho. No entanto, em outras espécies, como o pacu, a frequência alimentar afetou diretamente a taxa de crescimento (Fiod et al., 2010). Como o desempenho do tambaqui não foi alterado no presente estudo, a taxa de crescimento se manteve igual para todos os tratamentos, estando estas variáveis correlacionadas.

Com relação à sobrevivência, esta também não apresentou diferença estatística, mesmo sendo numericamente inferior na frequência de oito vezes diária. Com o lambari-do-rabo-amarelo, Hayashi et al. (2004) observaram um efeito linear negativo em razão do aumento da frequência alimentar, o que levou os autores a inferirem tratar-se da disputa dos animais pelo alimento, que, em pouca quantidade, pode favorecer os dominantes, deixando os menos nutridos susceptíveis à mortalidade. Este fato também pode ter ocorrido no presente estudo.

A manutenção da glicemia foi eficiente, sem que houvesse efeito das frequências de alimentação. Esta tem sido um indicador dos estados nutricional, fisiológico, e em estudos relacionados ao estresse (Martins et al., 2002; Melo, 2004; Fagundes, 2009). O aumento da glicose sanguínea parece ser mais influenciado pela elevação dos carboidratos do que a frequência alimentar (Baldan, 2008) com a elevação dos níveis de carboidratos na dieta. Ao avaliarem o desempenho de tambaquis cultivados sob diferentes densidades de estocagem e sob diferentes taxas de alimentação em tanques-rede, Chagas et al. (2005) também não encontraram efeito significativo sobre a glicemia. Este é um parâmetro simples de avaliação, de baixo custo, quando comparado a outras análises metabólicas, sem que seja necessário o sacrifício do animal, e permite avaliar o estado de higidez dos animais, seja em razão de distúrbios nutricionais ou de problemas com o manejo.

\section{CONCLUSÕES}

Frequências alimentares maiores não são recomendadas, por demandarem maior mão de obra, portanto duas alimentações diárias para o tambaqui são suficientes para manter os parâmetros de desempenho e fisiológico avaliados. A frequência alimentar não afetou o desempenho do tambaqui no período estudado. 


\section{REFERÊNCIAS}

ANDRADE, R.L.B.; WAGNER, R.L.; MAHI, I.; MARTINS, R.S. Custos de produção de tilápias (Oreochromis niloticus) em um modelo de propriedade da região oeste do Estado do Paraná, Brasil. Cienc. Rural, v.35, p.198-203, 2005.

BALDAN, A.P. Avaliação da tolerância do pacu (Piaractus mesopotamicus) a carboidratos. Jaboticabal. 102f. 2008. Tese (Doutorado em Aquicultura) - Pós-graduação em Aquicultura, Universidade Estadual Paulista - Centro de Aquicultura da Unesp - CAUNESP. Jaboticabal, SP, 2008.

CARNEIRO, P.C.F.; MIKOS, J.D. Frequência alimentar e crescimento de alevinos de Jundiá, Rhamdia quelen. Cienc. Rural, v.35, p.187-191, 2005.

CHAGAS, E.C.; GOMES, L.C.; MARTINS JÚNIOR, H. et al. Desempenho de tambaqui cultivado em tanques-rede, em lago de várzea, sob diferentes taxas de alimentação. Pesq. Agropec. Brasil, v.40, p.833-835, 2005.

CHAGAS, E.C.; GOMES, L.C.; MARTINS JÚNIOR, H.; ROUBACH, R. Produtividade de tambaqui criado em tanque-rede com diferentes taxas de alimentação. Cienc. Rural, v.37, p.11091115, 2007.

CORRÊA, C.F.; LEONARDO, A.F.G.; TACHIBANA, L.; CORRÊA-JÚNIOR, L. Frequência alimentar para juvenis de robalo-peva criados em água doce. Rev. Acadêm. Cienc. Agrar. Ambient., v.8, p.429-436, 2010.

CORRÊA, R.O.; TEIXEIRA, R.N.G.; FONSECA, V.S.; ALBUQUERQUE, F.E.A. Frequência alimentar de juvenis de tambaqui, Colossoma macropomum (Cuvier, 1818), cultivados emtanques - rede. Comunicado Técnico 221. EMBRAPA Amazônia Oriental. Belém-PA, 2009.

FERREIRA, R.A.; THIESEN, R.; COSTA, T.R. et al. Desempenho produtivo de alevinos de dourado (Salminus brasiliensis) submetidos a diferentes frequências de alimentação. Ensaios $e$ Ciências, v.11, p.33-38, 2007.
FIOD, M.S.R.; DUCATI, C.; CABRAL, M.C. et al. Efeito da frequência alimentar sobre o crescimento e a composição isotópica $(\delta 13 \mathrm{c}$ e $\delta$ 15n) de juvenis de pacu Piaractus mesopotamicus. Nucleus Animalium, v.2, p.41$52,2010$.

FRASCÁ-SCORVO, C.M. Comportamento alimentar do matrinxã brycon cephalus (Günther, 1869) em tanques de cultivo. 1999. $76 f$. Dissertação (Mestrado em Aquicultura) CAUNESP, Universidade Estadual, JaboticabalSP, 1999.

GRAEFF, À.; PRUNER, E.N. Variação percentual e frequência de alimento fornecido no desenvolvimento final de jundiás (Rhamdia quelen) na fase de recria. Associac. Latinoam. Produc. Anim., v.17, p.1-7, 2008.

HAYASHI, C.; MEURER, F.; BOSCOLO, W.R. Frequência de arraçoamento para alevinos de lambari do rabo-amarelo (Astyanax bimaculatus). Rev. Brasil. Zootec., v.33, p.21-26, 2004.

KUBITZA, F. Nutrição e alimentação de peixes. Piracicaba: Escola Superior de Agricultura Luiz de Queiroz, 1997. 74p.

KUBITZA, F.A versatilidade do sal na Piscicultura. Rev. Panor. Aquicul., v.1, p.14-23, 2007.

LUZ, R.K.; PORTELLA, M.C. Frequência alimentar na larvicultura de trairão (Hoplias lacerdae). Rev. Brasil. de Zootec., v.34, p.1442 1448, 2005.

MARTINS, M.L.; MORAES, F.R.; FUJIMOTO, R.Y. et al. Respostas do híbrido tambacu (Piaractus mesopotamicus Holmberg, 1887 macho x Colossoma macropomum Cuvier, 1818 fêmea) a estímulos simples ou consecutivo de captura. Bol. Instit. Pesca, v.28, p.195-204, 2002.

MEER, M.B.VAN DER.; HERWAARDEN, V.; VERDEGEM, M.C.J. Effect of number of meals and frequency of feeding on voluntary feed intake of Colossoma macropomum (Cuvier). Aquac. Resear., v.28, p.419-432, 1997.

MELO, F.B. Digestão e metabolismo de jundiá (Rhamdia quelen) submetido a diferentes regimes alimentares, 2004. 80f. Tese (Doutorado em Ciências fisiológicas) Universidade Federal de São Carlos, SP. 
MELO, L.A.S.; PEREIRA FILHO, M.; IZIEL, A.C.U.; STORTI FILHO, A. Avaliação econômica de um cultivo de tambaqui, Colossoma macropomum, na Amazônia ocidental. In: Reunião da SBZ. 15., 1998, Botucatu. Anais..., Botucatu/SP, p.38-41. 1998.

NDOME, C.B.; EKWU, A.O; ATEB, A.A. Effect of Feeding Frequency on Feed Consumption, Growth and Feed Conversion of Clarias gariepinus X Heterobranchus longifilis Hybrids American-Eurasian. J. Scient. Resear., v.6, p.6-12, 2011.

NUNES, E.S.; CAVERO, B.A.; PEREIRAFILHO, M.; ROUBACH, R. Enzimas digestivas exógenas na alimentação de juvenis de tambaqui. Pesq. Agropec. Brasil., v.4, p.139-143, 2006.

PAUlA, F.G. Desempenho do Tambaqui (Colossoma macropomum), da Pirapitinga (Piaractus brachypomum), e do híbrido Tambatinga (C. macropomum $\mathrm{x}$ Piaractus brachypomum) mantidos em viveiros fertilizados na fase de engorda. 2009. 57f. Dissertação (Mestrado em Ciência Animal) Escola de Veterinária, Universidade Federal de Goiás, Goiânia-GO. 2009.

SAMPAIO, L.A.; OLIVEIRA, M.; TESSER, M.B. Produção de larvas e juvenis do Peixe-rei marinho Odontesthes argentinensis submetidos à diferentes frequências alimentares. Rev. Bras. Agroc., v.13, p.271-274, 2007.
SANTOS, L.; FILHO, M.P.; SOBREIRA, C. et al. Exigência Proteica de juvenis de tambaqui (Colossoma macropomum) após privação alimentar. Acta Amazônica, v.40, p.597-604, 2010.

SILVA, C.R.; GOMES, L.C.; BRANDÃO, F.R. Effect of feeding rate and frequency on tambaqui (Colossoma macropomum) growth, production and feeding costs during the first growth phase in cages. Aquaculture, v.264, p.135-139, 2007.

SIPAÚBA-TAVARES, L.H. Limnologia Aplicada à Aquicultura. Jaboticabal: FUNEP, 1995. 70p.

SOUSA, R.M.R.; AGOSTINHO, C.A.; OLIVEIRA, F.A.; ARGENTIM, D.; NOVELLI, P.K.; AGOSTINHO, S.M.M. Productive performance of Nile tilapia (Oreochromis niloticus) fed at different frequencies and periods with automatic dispenser. Arq. Bras. Med. Vet. Zootec., v.64, p.192-197, 2012.

VAL, A.L.; ROLIM, P.R.; RABELO, H. Situação atual da aquicultura na região norte. In: VALENTI, W.C.; POLI, C.R.; PEREIRA, J.A.; BORGHETTI, J.R. (Ed.) 2000. Aquicultura no Brasil: bases para um desenvolvimento sustentável. Brasília: CNPq/MCT. 399p. 